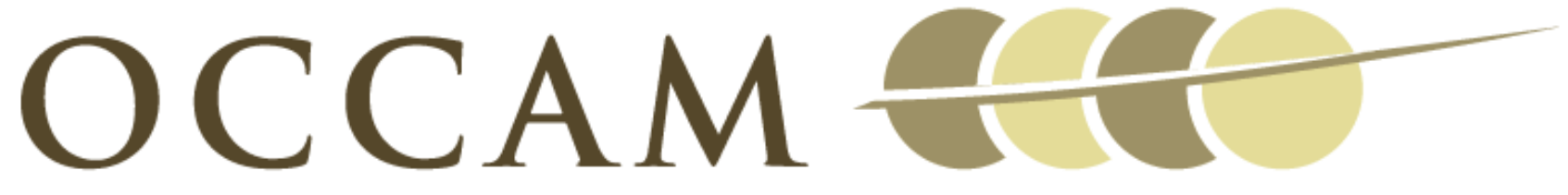

OXFORD CENTRE FOR COLLABORATIVE APPLIED MATHEMATICS

Report Number 10/62

Adsorption and desorption dynamics of citric acid anions in soil by

E. Oburger, D. Leitner, D. Jones, K. Zygalakis, A. Schnepf, T. Roose

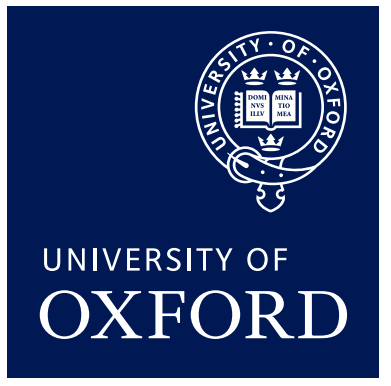

Oxford Centre for Collaborative Applied Mathematics Mathematical Institute 24 - 29 St Giles'

Oxford

OX1 3LB

England 

Adsorption and desorption dynamics of citric acid anions in soil

\begin{tabular}{|c|c|}
\hline Journal: & European Journal of Soil Science \\
\hline Manuscript ID: & EJSS-251-10 \\
\hline Manuscript Type: & Original Manuscript \\
\hline $\begin{array}{r}\text { Date Submitted by the } \\
\text { Author: }\end{array}$ & $25-O c t-2010$ \\
\hline Complete List of Authors: & $\begin{array}{l}\text { Oburger, Eva; BOKU - University of Natural Resources and Life } \\
\text { Sciences, Department of Forest and Soil Sciences } \\
\text { Leitner, Daniel; BOKU - University of Natural Resources and Life } \\
\text { Sciences, Department of Forest and Soil Sciences } \\
\text { jones, davey; university of wales, SAFS } \\
\text { Zygalakis, Konstantinos; University of Oxford, Mathematical } \\
\text { Institute } \\
\text { Schnepf, Andrea; BOKU - University of Natural Resources and Life } \\
\text { Sciences, Vienna, Department of Forest and Soil Sciences } \\
\text { Roose, Tiina; University of Oxford, Mathematical Institute }\end{array}$ \\
\hline Keywords: & $\begin{array}{l}\text { carboxylic acid, citric acid, freundlich isotherm, mathematical } \\
\text { model, rhizosphere, hysteresis }\end{array}$ \\
\hline
\end{tabular}




\section{Adsorption and desorption dynamics of citric acid anions in soil}

2 E. Oburger ${ }^{\text {a }}$, D. Leitner ${ }^{\text {a }}$, D.L. Jones ${ }^{\text {b }}$, K.C. Zygalakis ${ }^{\text {c }}$, A. Schnepf ${ }^{\text {a, }}{ }^{*}$, T. Roose ${ }^{\text {d }}$

3

$4 \quad{ }^{a}$ Department of Forest and Soil Sciences, BOKU - University of Natural Resources and Life

5 Sciences, A-1190 Vienna, Austria

$6 \quad{ }^{\mathrm{b}}$ Environment Centre Wales, Bangor University, Gwynedd, LL57 2UW, UK

$7 \quad{ }^{\mathrm{c}}$ Oxford Centre for Collaborative Applied Mathematics, Mathematical Institute, University of

$8 \quad$ Oxford, OX1 2RB, Oxford UK

$9 \quad{ }^{\mathrm{d}}$ Bioengineering Group, Faculty of Engineering and Environment, University of

10 Southampton, Southampton, SO17 1BJ. UK

11

12 *Corresponding author: Tel.: +43 147654 3126; fax: +43 1476543130.

13 E-mail address: andrea.schnepf@boku.ac.at (Andrea Schnepf).

14 
15

16

17

18

19

\section{Summary}

The functional role of organic acid anions (e.g. citrate, oxalate, malonate, etc) in soil has been intensively investigated with special focus either on (i) microbial respiration and soil carbon dynamics, (ii) nutrient solubilization, or (iii) metal detoxification. Considering the potential impact of sorption processes on the functional significance of these effects, comparatively little is known about the adsorption and desorption dynamics of organic acid anions in soils. The aim of this study therefore was to experimentally characterize the adsorption and desorption dynamics of organic acid anions in different soils using citrate as a model carboxylate. Results showed that both adsorption and desorption processes were fast, reaching a steady state equilibrium solution concentration within approximately 1 hour. However, for a given total soil citrate concentration $\left(c_{\text {tot }}\right)$ the steady state value obtained was critically dependent on the starting conditions of the experiment (i.e. whether most of the citrate was initially present in solution $\left(c_{1}\right)$ or held on the solid phase $\left(c_{\mathrm{s}}\right)$ ). Specifically, desorption-led processes resulted in significantly lower equilibrium solution concentrations than adsorptionled processes indicating time-dependent sorption hysteresis. As it is not possible to experimentally distinguish between different sorption pools in soil (i.e. fast, slow, irreversible adsorption/desorption), a new dynamic hysteresis model was developed that relies only on measured soil solution concentrations. The model satisfactorily explained experimental data and was able to predict dynamic adsorption and desorption behaviour. To demonstrate its use we applied the model to two relevant scenarios (exudation and microbial degradation), where the dynamic sorption behaviour of citrate occurs. Overall, this study highlights the complex nature of citrate sorption in soil and concludes that existing models need to incorporate both a temporal and sorption hysteresis component to realistically describe the role and fate of organic acids in soil processes. 
Keywords: Carboxylic acid, Citric acid, Freundlich isotherm, Mathematical model, Rhizosphere, Hysteresis

\section{Introduction}

Low molecular weight (LMW) organic acid anions play an important role in many soil biogeochemical processes as they positively influence the growth of plants and microorganisms by enhancing nutrient solubility (e.g. P, Fe, Zn) and by complexing and detoxifying metals present in high concentrations (e.g. Al, Cd, Zn; Neumann et al., 1999; Dakora and Phillips, 2002; Ryan et al., 2001). In addition, organic acid anions provide the soil microbial community with a relatively labile source of carbon $(\mathrm{C})$ which promotes microbial activity and growth (Jones, 1998). Due to rapid removal processes from soil solution (i.e. sorption and biodegradation), organic acid anions typically only constitute a minor fraction of the dissolved organic carbon (DOC) in soil ( $<10 \%$ of total DOC; van Hees et al., 2002). Nevertheless, they have been found to make an important contribution to total soil respiration, with $20-75 \%$ of total soil respiration being potentially derived from organic acid breakdown (van Hees et al., 2008).

Whilst small amounts of carboxylic acids can enter soil from precipitation, organic wastes and as a by-product of soil organic matter (SOM) mineralization, it is assumed that active excretion and passive exudation by living plant roots and microorganisms represents their main route of entry into soil (Jones et al., 2004; van Hees et al., 2008). Furthermore, increased exudation rates are often observed as a metabolic response to environmental stress (Hoffland et al., 1989; Zhang et al., 1997; Ryan et al., 1995). Ultimately, this is expected to lead to localized hotspots of organic acid activity in soil and both spatially and temporally heterogeneous concentration patterns.

Due to their proposed role in pedogenic processes, the fate of organic acids has been intensively studied in plant-microbial-soil systems. To date, the main focus of investigations 
has either been on (i) organic acid mineralization patterns and their role in soil $\mathrm{C}$ dynamics (van Hees et al., 2008; Oburger et al., 2009), (ii) the role of organic acids in nutrient solubilization (mainly P; e.g. Palomo et al., 2006), or (iii) organic acids as an $\mathrm{Al}^{3+}$ detoxification mechanism (e.g. Ma, 2005). In all these different aspects, the sorption (i.e. adsorption and desorption) behaviour of organic acid anions plays a key role, as it will ultimately determine their movement, residence time, availability and functional effect in soil (Jones et al., 1996a). While adsorption of organic acid anions has been intensively studied in a range of soils (Jones and Brassington, 1998; Ström et al., 2001; van Hees et al., 2003, Oburger et al., 2009), comparatively little information is available on their desorption dynamics. Traditionally, it has been assumed that sorption of organic acid anions, like that of other anions in soil (e.g. $\mathrm{SO}_{4}{ }^{2-}$ ) is fully reversible and therefore sorption can simply be described by a single soil-solution partition coefficient (Darrah, 1991). Whether organic acid adsorption is fully reversible, however, remains critical in regulating the effective zone of influence around exuding organisms. In a recent study, Strahm and Harrison (2008) observed that at low $\mathrm{pH}$ very little malonate could be desorbed from a variable charge soil in a sequential desorption approach and that decreased biodegradation rates correlated well with these reduced desorption patterns.

Due to the lack of available techniques to experimentally measure organic acid anion gradients in soil at sufficient spatial resolution (e.g. diffusion away from roots), mathematical models are often used to predict organic acid movement in soil (Jones et al., 1996b; Kirk et al., 1999). As some organic acid anions (e.g. oxalate, citrate) may strongly adsorb to the soil's solid phase (e.g. oxalate, citrate), it is critical to accurately describe sorption processes that represent a key control on the diffusional flux. Parametrisation of kinetic sorption models, however, is difficult, since the number of model parameters are generally high and initial conditions can sometimes not be obtained by experimental measurements (e.g. differentiation of the sorbed concentration in a slow and in a fast pool). There are several different modelling 
approaches. The simplest approach is to use a time dependent explicit empirical equation (e.g. an extension to the Freundlich equation) that is fitted to kinetic sorption data (Barrow, 2008). Another approach uses multi-reaction models, which describe the reaction by ordinary differential equations using different pools with dissimilar reaction rates for the sorbed ion (e.g. a slow and a fast pool) (Selim and Zhu, 1997; Barrow et al., 2000). Finally, more complex models include diffusion into the matrix of the adsorbing material (Tsakiroglou and Ioannidis, 2008; Ptashnyk et al., 2010; Ptashnyk and Roose, 2010).

The aim of this study was to further elucidate the fate of organic acid anions in soil with special regard to sorption processes. We experimentally characterized the sorption behaviour of organic acids, by carrying out adsorption and desorption experiments under sterile conditions in 3 different agricultural soils using citrate as a model carboxylate. Experimental data was then used to develop a new kinetic sorption model that is able to predict the concentration of citrate in the soil solution over time.

\section{Materials and Methods}

Soils

Three agricultural soils were used in the study. Soils, sampling sites, and soil parameter determination procedure have been described in detail in Oburger et al. (2009). Briefly, a Eutric Cambisol topsoil (Ah horizon, 0-15 cm depth) and a Haplic Podzol topsoil (Ah horizon, 0-15 cm depth) and subsoil (Bs horizon, 20-40 cm depth) were collected at

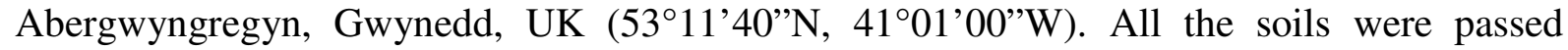
through a 4-mm sieve and stored field-moist at $4{ }^{\circ} \mathrm{C}$ until analysis. Prior to experimentation the soil was sterilized by heating $\left(30 \mathrm{~min}\right.$ at $\left.80^{\circ} \mathrm{C}\right)$ to prevent microbial degradation of the organic acids (Kuzyakov and Jones, 2006). The general properties of the soils are shown in Table 1. All treatments were carried out in duplicate unless otherwise stated. 


\section{Adsorption curves}

To determine the sorption behaviour of citrate in the different experimental soils, concentration-dependent adsorption envelopes were measured at a 1:10 (w/v) soil-to-solution ratio. Briefly, heat sterilized soil (1 g dry weight) was shaken with $10 \mathrm{ml}$ of $1,5,10,25,50$ and $100 \mu \mathrm{M}^{14} \mathrm{C}$-citrate $\left(0.3 \mathrm{kBq} \mathrm{g}^{-1}\right)$ for 1 hour at $20^{\circ} \mathrm{C}$ on a reciprocal shaker at $200 \mathrm{rev}^{-} \mathrm{min}^{-}$ 1. The background electrolyte in the citrate solutions was $1 \mathrm{mM} \mathrm{KCl}$. Subsequently, samples were centrifuged for $5 \mathrm{~min}$ at $18,000 \mathrm{~g}$ and the ${ }^{14} \mathrm{C}$-citrate present in the supernatant solution determined by liquid scintillation counting using Ultima Gold ${ }^{\mathrm{TM}}$ XR scintillation fluid (PerkinElmer Corp., Shelton, USA) and a 1900CA TRI-CARB ${ }^{\circledR}$ Liquid Scintillation analyzer (Canberra Industries Inc., Meriden, CT).

\section{Desorption curves}

Desorption behaviour of citrate in the 3 experimental soils was determined by using a parallel batch extract approach. Soil $(1 \mathrm{~g})$ was spiked with $0.25 \mathrm{ml}{ }^{14} \mathrm{C}$-labelled citrate $(0.5$ $\mu \mathrm{mol} \mathrm{g}$; $0.3 \mathrm{kBq} \mathrm{g}^{-1}$ ) and allowed to equilibrate for $30 \mathrm{~min}$ at $20^{\circ} \mathrm{C}$. At this point, $99 \%$ of the added ${ }^{14} \mathrm{C}$-citrate was adsorbed to the solid phase (data not presented). Subsequently, different volumes of $\mathrm{KCl}(1 \mathrm{mM})$ were added to the experimental soils resulting in final soil-tosolution ratios $(\mathrm{w} / \mathrm{v})$ of $1: 1,1: 2.5,1: 5,1: 10,1: 25,1: 50,1: 75$, and 1: 100. The background solution of $1 \mathrm{mM} \mathrm{KCl}$ was chosen as it does not significantly interact with citrate. Samples were then shaken for 1 hour at $200 \mathrm{rev} \min ^{-1}$, centrifuged $(18,000 \mathrm{~g}, 5 \mathrm{~min})$ and the supernatant recovered. The amount of ${ }^{14} \mathrm{C}$-citrate desorbed into solution was determined by liquid scintillation counting as described above.

\section{Adsorption kinetics}

The adsorption kinetics of citrate to each soil were determined by measuring the change in concentration of ${ }^{14} \mathrm{C}$-citrate in the soil solution over time, after the addition of $0.5 \mu \mathrm{mol} \mathrm{g}{ }^{-1}$ 
144 citrate soil. Briefly, $10 \mathrm{ml}$ of ${ }^{14} \mathrm{C}$-labelled citrate $\left(0.3 \mathrm{kBq} \mathrm{g}^{-1}, 50 \mu \mathrm{M}\right)$ was added to $1 \mathrm{~g}$ of soil 145 and the soil shaken as described above. At different times (1, 2, 5, 10, 30, 60, 120, 240 and $146480 \mathrm{~min}$ ) after ${ }^{14} \mathrm{C}$-citrate addition, $100 \mu \mathrm{l}$ of the soil suspension was removed, centrifuged 147 and the ${ }^{14} \mathrm{C}$ remaining in solution determined as described above. These times were chosen to 148 reflect expected residence times of organic acid anions in the rhizosphere (Ryan et al., 2001).

Desorption kinetics

For the desorption kinetics, soil $(1 \mathrm{~g})$ was incubated for $30 \mathrm{~min}$ with ${ }^{14} \mathrm{C}$-citrate $(0.5$ $\mu \mathrm{mol} \mathrm{g} \mathrm{g}^{-1} ; 0.3 \mathrm{kBq}$ ) and then shaken with $10 \mathrm{ml}$ of $1 \mathrm{mM} \mathrm{KCl}$ at $200 \mathrm{rev} \min ^{-1}$. At different times $(1,2,5,10,30,60,120,240$ and $480 \mathrm{~min})$ after $\mathrm{KCl}$ addition $100 \mu \mathrm{l}$ of the soil

154 suspension was removed, centrifuged and the ${ }^{14} \mathrm{C}$ remaining in solution determined as 155 described above.

\section{Results and discussion}

Experimental results

The concentration and time-dependent citrate adsorption and desorption isotherms are

160 presented in Figure 1. As expected, significant differences in sorption behaviour were observed in the three soils. In general, citrate adsorption increased with increasing amounts of anionic binding sites in the soils (Cambisol $<$ Podzol Ah $<$ Podzol Bs, Table 1) while citrate desorption did not follow the same trend (Podzol Ah > Cambisol > Podzol Bs). Citrate

164 adsorption and desorption reactions were both fast, approaching steady state after 165 approximately 1 hour (Fig. 1bc) within the experimental measurement period (see also kinetic data in Fig. 1a). This result implies that sorption processes of citrate are almost instantaneous, highly soil-type specific and dependent on carboxylate concentration. However, depending on

168 the initial conditions (i.e. either all the citrate initially in solution as in the adsorption 169 experiments or all on the solid phase as in the desorption experiments) the forward 
170 (adsorption) and backward (desorption) reactions reached a different steady state during the

171 experimental time period. This resulted in markedly different shapes for the adsorption and

172 desorption curves in each soil, clearly indicating sorption hysteresis (Fig. 1a). Within a short

173 time period $(8 \mathrm{~h})$, only a small proportion of the adsorbed citrate was readily desorbable (Fig.

174 1c). This desorption potential was also soil type dependent and represented $28 \pm 2 \%$ of that 175 initially held on the solid phase in the Cambisol, $44 \pm 3 \%$ in the Podzol Ah and $23 \pm 1 \%$ in the

176 Podzol Bs. We therefore propose that the release of adsorbed citrate can be described by a 177 two-pool model with a fast-release (labile) pool and a slow-release (fixed) pool of which the

178 latter does not seem to influence the short-term sorption steady state. Our results, however, do

179 not allow us to make any conclusions about whether this fixed pool represents truly

180 irreversible sorption by the solid phase. Evidence presented in microbial biodegradation

181 studies of organic acid anions in soils indicate that where mineralization is relatively rapid,

182 irreversible sorption is unlikely (Ström et al., 2001; van Hees et al., 2003; Oburger et al., 183 2009ab).

Sorption hysteresis and the fate of citrate in soil

186 Sorption hysteresis is a frequently observed phenomenon that occurs when distinct discrepancies between adsorption and desorption curves are found (Zhang and Selim, 2005). True hysteresis in soils has been reported for inorganic and organic compounds, as a consequence of slow precipitation at the mineral surface and/or slow diffusion to sites within

190 the soil matrix resulting in irreversible retention of the solute (Barrow, 1984; Huang et al., 191 1997; Zhang and Selim, 2005). Experimentally, solid-solute contact time is a crucial factor, as 192 observed hysteresis can often be attributed to the failure of attaining true equilibrium during 193 either or both sorption and desorption procedures (Lesan and Bhandari, 2003). Adsorption 194 and desorption processes of highly reactive anions like phosphate or arsenate have been found 195 to continue for weeks and even years (O'Reilley et al., 2001; Raven et al., 1998), but the same 
196 authors also concluded that the majority of sorption reactions occurred within the first 2 to 24

197 h. In many longer-term sorption studies using non-sterile soil, however, microbial 198 immobilization of the added anions is also likely to occur. If these anions are subsequently 199 transformed and incorporated into the microbial community (e.g. $\mathrm{PO}_{4}{ }^{3-}$ into microbial lipids), 200 based on known microbial biomass turnover times (ca. 1-3 months) they would appear to be 201 irreversibly fixed. In this study we used sterile soil to remove this potential artefact.

In contrast to inorganic nutrients or recalcitrant organic pollutants, where a better knowledge of long-term sorption behaviour helps improving soil management practices, longtime sorption experiments with low-molecular-weight carboxylates will only provide a partial view of their fate due to the presence of other highly dynamic soil processes (e.g. organic acid input via exudation and removal by rapid mineralization). The average half-life of $\mathrm{C}$ derived

207 from citrate in soil typically ranges from 2-20 d (Oburger et al., 2009b), however, primary microbial removal of organic acids from the soil solution has an even shorter half life in the region of 0.5-12 h (Oburger et al., 2009ab). Considering these highly dynamic processes, short-term sorption reactions are particularly important since they will determine the 211 instantaneous solid-solution partitioning of organic anions. The question arises about how and 212 to what extent do sorption processes influence the bioavailability of these carboxylates, and 213 therefore their potential functional effect in soil (e.g. metal detoxification, nutrient 214 solubilization, soil respiration). Previous observations of reduced microbial degradation of 215 organic acid anions in strongly sorbing soils indicate that their bioavailability is controlled by 216 sorption processes (Ström et al., 2001; van Hees et al., 2003; Oburger et al., 2009a). The 217 distribution kinetics of organic acid anions between the soil solution, soil matrix, and microbial biomass, however, still remain unknown. 


\section{Interpretation of experimental results with a new kinetic sorption model}

The different shape of the adsorption and desorption curve indicates that in the experimental timescales used here there are two different equilibria for the forward and backward reaction. Here we aim at developing a model that represents this hysteresis effect and accurately describes sorption dynamics. The model describes all the experimental data by including the steady-states of both citrate adsorption and desorption. The simplest dynamic mathematical model that can describe the observed hysteresis effect is a kinetic model with three steady-states (two stable and one unstable), i.e., a model of the form

$$
\frac{d q}{d t}=f_{a d}\left(c_{l}, s s r\right) \cdot f_{u s}\left(c_{l}, s s r\right) \cdot f_{d e}\left(c_{l}, s s r\right)
$$

where, at equilibrium, $f_{a d}$ becomes the steady-state that corresponds to the adsorption isotherm, $f_{d e}$ becomes the steady-state that corresponds to the desorption isotherm and because there has to be one unstable isotherm between two stable ones we include $f_{u s}$ as an unknown (not measured) unstable steady-state.

If Freundlich equations are used, the functions $f_{a d}$ and $f_{d e}$ are given by

$$
f_{a d}=\frac{r}{s s r}\left(c_{t}-s s r \cdot c_{l}\right)-k_{a d} \cdot c_{l}^{n}{ }^{a d}
$$

and

$$
f_{d e}=\frac{r}{s s r}\left(c_{t}-s s r \cdot c_{l}\right)-k_{d e} \cdot c_{l}^{n} d e,
$$

where $r$ represents the reaction rate constant, $s s r$ is the solution soil ratio $\left(\mathrm{g} \mathrm{g}^{-1}\right), c_{t}$ is the total concentration in soil $\left(\mu \mathrm{mol} \mathrm{g} \mathrm{g}^{-1}\right), c_{l}$ is the concentration of citrate in soil solution $(\mu \mathrm{M})$, $k$ and $n$ are the Freundlich equation parameters, and the superscripts $a d$ and $d e$ indicate the Freundlich parameters of the ad- and desorption isotherms, respectively. According to the experimental setup, $c_{s}$ and $c_{l}$ are dependent, i.e. $c_{s}=c_{t}-s s r \cdot c_{l}$. Therefore in Eqs. (2) and (3), the term $c_{t}-s s r \cdot c_{l}$ is used instead of $c_{s}$, where $c_{s}$ is the concentration of adsorbed 
245 citrate $\left(\mu \mathrm{mol} \mathrm{g}{ }^{-1}\right)$. The function $f_{u s}\left(c_{l}, s s r\right)$ represents the unstable steady state which must 246 lie between the two stable steady states. A simple functional form of $f_{u s}$ can be obtained 247 interpolating between the $f_{a d}$ and $f_{d e}$ :

$$
f_{u s}:=\theta \cdot f_{a d}+(1-\theta) \cdot f_{d e},
$$

249 where $\theta$ is a value between 0 and 1 .

In Figure 2 we illustrate this approach using a phase protrait, in which the three lines represent three steady states of the system. The arrows indicate the direction in which the model state $\left(c_{s}, c_{l}\right)$ develops for both the ad- and desorption kinetic experiments. For adsorption scenarios, we start at the $x$-axis and, at equilibrium, the lower stable steady-state is reached. For desorption scenarios, we start at the $y$-axis and the upper stable steady-state is reached. In between the two stable steady-states, there is an unstable steady state. Depending on the starting point with respect to the unstable isotherm adsorption (Fig. 2, areas B and D) or desorption will occur (Fig. 2, areas A and C).

Fitting the model to the experimental data

The kinetic sorption model given by Eqns (8)-(11) has six parameters: two Freundlich parameters for adsorption $\left(k_{a d}, n_{a d}\right)$, two Freundlich parameters for desorption $\left(k_{d e}, n_{d e}\right)$, the rate $r$ and the value $\theta$. In a first step, the Freundlich parameters $k_{a d}, k_{d e}, n_{a d}$ and $n_{d e}$ are obtained by fitting two Freundlich isotherms to the ad- and desorption isotherm data. These parameters are used in the second step as initial parameters for fitting the full model.

When fitting the Freundlich parameters to experimental data, it must be considered that $c_{s}$ 266 and $c_{l}$ are dependent so that a measurement error of $c_{l}$ will result in an error of $c_{s}$. 267 Therefore, the squared distance between an observed data point $\left(c_{s, i}, c_{l, i}\right)$ and the Freundlich sorption curve is obtained by first evaluating $c_{l, \mathrm{i}}$ from the implicit equation 


$$
c_{t}-s s r \cdot c_{l, i}^{*}=k_{f} \cdot c_{l, i}^{* n},
$$

270

The summed squared error sse is then given by

$$
s s e=\sum_{i=1}^{N} s s r^{2} \cdot\left(c_{l, i}^{*}-c_{l, i}\right)^{2}+\left(c_{s, i}^{*}-c_{s, i}\right)^{2}
$$

where $N$ is the number of observations. Minimization of Eqn (6) yields the Freundlich parameters $k$ and $n$. In the case of adsorption, ssr is constant and in the case of desorption, $c_{t}$ is constant.

Parameter estimation of the full kinetic model is performed by minimizing the summed squared error

$$
s s e=\sum_{i=1}^{N} s s r^{2} \cdot\left(c_{l, t_{i}}^{*}-c_{l, t_{i}}\right)^{2}+\left(c_{s, t_{i}}^{*}-c_{s, t_{i}}\right)^{2},
$$

where $c_{l, t_{i}}$ are the observed kinetic data at times $t_{i}$, and $c_{l, t_{i}}^{*}$ are the model results which are obtained by numerically integrating the ordinary differential equations (Eqns. 1-4) using the Matlab function ode15s. The sorbed concentrations are calculated from the total concentration: $c_{s, t_{i}}=c_{t}-s s r \cdot c_{l, t_{i}}$ and $c_{s, t_{i}}^{*}=c_{t}-s s r \cdot c_{l, t_{i}}^{*}$. The total summed squared error is obtained by adding up the errors of the ad- and desorption data (Eqn. 6) and the kinetic adand desorption data (Eqn. 7).

Figure 1 shows the model results matched to the experimentally observed isotherm and kinetic data. In Figure 1a, we see the model fits for the ad- and desorption isotherms of each experimental soil. Since $c_{s}$ and $c_{l}$ are dependent, the error bars of the data points are not vertical but have a slope. In the case of adsorption, the slope is $-s s r$, see Eqn (2). In the case of desorption, the slopes of the error bars are different for each observation $i$ and are given by $-s s r_{i}$, see Eqn (3). The kinetic data lie on the curve $c_{s}=c_{t}-s s r \cdot c_{l}$, which is represented by the dotted line (Fig. 1a). Figures $1 \mathrm{~b}$ and 1c show the dynamic behaviour of the system and the observed kinetic data. In the adsorption experiment, initially all the citrate is present in soil 
292

solution and none is present on the solid phase. In the desorption experiment, initially all the citrate is adsorbed to the solid phase and none is present in soil solution. The sorption parameters obtained from fitting the model are shown in Table 2.

This descriptive kinetic model represented the experimental data using parameters from the Freundlich isotherms. A big advantage of the approach presented here is its simplicity. In contrast to multi- pool approaches, the model depends only on one pool, the concentration in soil solution $c_{l}$, which can be measured. Therefore, the parameters of the model can be more easily fit while the model is still able to capture the hysteresis effect and the dynamic behaviour of the system. A drawback of the model is that mechanistic interpretation of underlying processes is not possible. However, the use of Freundlich isotherms makes it easy to interpret the parameters and to compare them to sorption parameters found in the literature (e.g. Ström et al., 2001).

In all soils, the adsorption isotherms had larger $n$ values and smaller $k$ values than in the desorption isotherm. This means that at low soil solution concentrations the soil-solution-tosolid phase partition coefficient $\left(K_{\mathrm{d}}\right)$ values are higher for the desorption isotherms than for the adsorption isotherm. However, with increasing soil solution concentration the $K_{\mathrm{d}}$ value of the desorption isotherm decreases stronger than in the case of adsorption. The parameter $r$ describes the speed at which the states reach their equilibrium. Overall, the sorption rate was lower in the Cambisol in comparison to both Podzol horizons. The parameter $\theta$ determines the position of the unstable steady state (see Fig. 2 and Eqn. 4). In all soils $\theta$ was very small (Table 2), thus we deduce that the unstable steady state lies close to the desorption isotherm, and area $\mathrm{C}$ increases, while area $\mathrm{B}$ becomes vanishingly small (Fig. 2).

\section{Modelling the sorption behaviour of citrate in soil - a theoretical case study}

The model developed here was used to predict the solid-solution partitioning of citrate in one of the experimental soils (Podzol Bs) for two different but common scenarios in soil 
318 (the pulse input of citrate by root exudation and the subsequent rapid microbial degradation of

319 citrate after exudation ceases). The initial condition for both scenarios was an assumed root 320 exudation event resulting in a citrate soil solution concentration of $40 \mu \mathrm{M}$ (Jones et al., 321 1996b). In scenario 1 (Fig. 3) a consecutive exudation event occurred after 80 min, increasing 322 the citrate solution concentration after the first exudation event (steady state I $c_{l 0}$ ) by another $32315 \mu \mathrm{M}\left(c_{l 1}\right)$. The model reacts according to the changes in the system and predicts a new, 324 adapted solid-solution partitioning of citrate (steady state II $c_{l l}$, Fig. 3a). The time course of 325 the changes in citrate soil solution concentration is shown in Figure 3b. In the second scenario 326 (Fig. 4), rapid microbial degradation (no time dynamics of the mineralization process were 327 considered) decreases the remaining soil solution concentration (steady state $I c_{l 0}$ ) and 328 therefore induces citrate desorption until a new steady state (steady state II $c_{l 1}$ ) is reached. 329 Figure $4 \mathrm{~b}$ shows the dynamic sorption behaviour of citrate before and after microbial 330 degradation.

\section{Conclusions and outlook}

Adsorption processes are known to strongly affect the bioavailability of organic acid

334 anions (e.g. citrate, malonate, oxalate, etc.) in soil, however, relatively little is known about 335 the dynamics of this process or its reversibility (desorption). Our results show that under 336 sterile conditions, both adsorption and desorption reactions reached a plateau after 337 approximately $1 \mathrm{~h}$ in all soils, but the steady state solution concentrations of the forward and 338 backward reactions differed significantly and sorption hysteresis of citrate was observed in all

339 three soils within the period of time investigated here. A descriptive mathematical model was 340 developed that was able to predict the change in citrate soil solution concentration in relation 341 to dynamic sorption processes and captured the experimentally observed hysteresis effect. In 342 contrast to multi-pool approaches, the model presented here depends only on measured citrate 
343 concentrations in soil solution. A drawback of the model is that mechanistic interpretation of 344 the underlying processes is not possible.

Our results highlight the impact that the dynamics of adsorption and desorption of

346 organic acid anions will have on their bioavailability and functional role in soil. In non-sterile 347 soils, it is impossible to experimentally distinguish between adsorption and microbial uptake, 348 as only the change in soil solution concentration can be determined. We propose that the 349 dynamic sorption model developed here could be combined with experimentally derived 350 mineralization data to predict the fate of citrate-derived $\mathrm{C}$ over time in non-sterile soil. 351 Ultimately, this will prove useful in the development of more robust mathematical models to 352 describe organic acid fate in soil (e.g. in the rhizosphere, mycosphere, pedogenesis etc).

\section{Acknowledgements}

This work was supported by the Vienna Science and Technology Fund (WWTF, Grant

No.: MA07-008), the Austrian Science Fund (FWF, Grant No.: P20069) and the UK

Biotechnology and Biological Sciences Research Council. K.C. Zygalakis was supported by Award No. KUKC1-013-04, made by King Abdullah University of Science and Technology (KAUST). Andrea Schnepf is a Hertha-Firnberg Research Fellow. Tiina Roose is a Royal Society University Research Fellow.

\section{References}

Altfelder S., Streck T., Richter J. 2000. Nonsingular sorption of organic compounds in soil: the role of slow kinetics. Journal of Environmental Quality 29, 917-925.

Barrow N.J. 1978. The description of phosphate adsorption curves. Journal of Soil Science 29, 447-462.

Barrow N.J. 1984. A mechanistic model for describing the sorption and desorption of phosphate by soil. Journal of Soil Science 34, 733-750. 
Barrow N.J. 2008. The description of sorption curves. European Journal of Soil Science 59, 900-910.

Barrow N.J., Hansen H.C.B., Hansen P.E., Magid J. 2000. A note on the description of the kinetics of phosphate sorption. European Journal of Soil Science 51, 531-535.

Dakora F.D., Phillips D.A. 2002. Root exudates as mediators for mineral acquisition in lownutrient environments. Plant and Soil 245, 35-47.

Darrah P.R. 1991. Measuring the diffusion-coefficients or rhizosphere exudates in soil. 2. The diffusion of sorbing compounds. Journal of Soil Science 42, 421-434.

Gerke J., Beißner L., Römer W. 2000. The quantitative effect of chemical phosphate mobilization by carboxylate anions on $\mathrm{P}$ uptake by a single root. I. The basic concept and determination of soil parameters. Journal of Plant Nutrition and Soil Science 163, 207-212.

Hoffland E., Findenegg G.R., Nelemans J.A. 1989. Solubilization of rock phosphate by rape II. Local root exudation of organic acids as a response to P-starvation. Plant and Soil 113, 161-165.

Huang W., Weber Jr.W.J. 1997. A Distributed reactivity model for sorption by soils and sediments. 10. relationships between desorption, hysteresis, and the chemical characteristics of organic domains. Environmental Science \& Technology 31, 25622569.

Huang W., Yu H., Weber Jr.W.J. 1998. Hysteresis in the sorption and desorption of hydrophobic organic contaminants by soils and sediments. 1. A comparative analysis of experimental protocols. Journal of Contaminant Hydrology 31, 129-148.

Jones D.L. 1998. Organic acids in the rhizosphere - a critical review. Plant and Soil 205, 2544.

Jones D.L., Darrah P.R. 1994. Role of root derived organic-acids in the mobilization of nutrients from the rhizosphere. Plant and Soil 166, 247-245. 
395 Jones D.L. and Brassington D.S. 1998. Sorption of organic acids in acid soils and its 396 implication in the rhizosphere. European Journal of Soil Science 49, 447-455.

397 Jones D.L., Darrah P.R., Kochian L.V. 1996a. Critical evaluation of organic acid mediated 398 iron dissolution in the rhizosphere and its potential role in root iron uptake. Plant and Soil 180, 57-66.

Jones D.L., Prabowo A.M., Kochian L.V. 1996b. Aluminium-organic acid interactions in acid 401 soils II. Influence of solid phase sorption on organic acid-Al complexation and Al rhizotoxicity. Plant and Soil 182, 229-237.

Jones D.L., Hodge A., Kuzyakov Y. 2004. Plant and mycorrhizal regulation of 404 rhizodeposition. New Phytologist 163, 459-480.

Kirk G.J.D., Santos E.E., Santos M.B. 1999. Phosphate solubilization by organic anion 406 excretion from rice growing in aerobic soil: rates of excretion and decomposition, effects on rhizosphere $\mathrm{pH}$ and effects on phosphate solubility and uptake. New Phytologist 142, 185-200.

Kuzyakov Y., Jones D.L. 2006. Glucose uptake by maize roots and its transformation in the rhizosphere. Soil Biology \& Biochemistry 38, 851-860.

Lesan H.M., Alok Bhandari A. 2003. Atrazine sorption on surface soils: time-dependent phase distribution and apparent desorption hysteresis. Water Research 37, 1644-1654.

Ma J.F. 2005. Physiological mechanisms of Al resistance in higher plants. Soil Science and Plant Nutrition 51, 609-612.

Ma L., Selim H.M. 1997. Evaluation of nonequilibrium models for predicting atrazine transport in soils. Soil Science Society of America Journal 61, 1299-1307.

Neumann G., Römheld V. 1999. Root excretion of carboxylic acids and protons in phosphorous - deficient plants. Plant and Soil 211, 121-130. 
419 O'Reilly S.E., Strawn D.G., Sparks D.L. 2001. Residence time effects on arsenate adsorption/desorption mechanisms on goethite. Soil Science Society of America Journal 65, 67-77.

Oburger E., Kirk G.J.D., Wenzel W.W., Puschenreiter M., Jones D.L. 2009a. Interactive effects of organic acids in the rhizosphere. Soil Biology \& Biochemistry 41, 449-457.

Oburger E., Jones D.L. 2009b. Substrate mineralization studies in the laboratory show different microbial C partitioning dynamics than in the field. Soil Biology \& Biochemistry 41, 1951-1956.

Palomo L., Claassen N., Jones D.L. 2006. Differential mobilization of $\mathrm{P}$ in the maize rhizosphere by citric acid and potassium citrate. Soil Biology \& Biochemistry 38, 683692.

Ptashnyk M., Roose T., Kirk G.J.D. 2010. Diffusion of strongly sorbed solutes in soil: A dualporosity model allowing for slow access to sorption sites and time-dependent sorption reactions. European Journal of Soil Science 61, 108-119.

Ptashnyk M., Roose T. 2010. Derivation of the macroscopic model for transport of strongly sorbed solutes in the soil using homogenization theory. SIAM Journal of Applied Mathematics 70, 2097-2118.

Raven K.P., Jain A., Loeppert R.H. 1998. Arsenite and arsenate adsorption on ferrihydrite: Kinetics, equilibrium, and adsorption envelopes. Environmental Science \& Technology 32, 344-349.

Ryan P.R., Delhaize E, Randall P.J. 1995. Characterisation of Al-stimulated efflux of malate from the apices of Al-tolerant wheat roots. Planta 196, 103-10.

Ryan P.R., Delhaize E., Jones D.L. 2001. Function and mechanism of organic anion exudation from plant roots. Annual Review of Plant Physiology and Plant Molecular Biology 52, 527-560. 
444 Selim H.M., Zhu H. 2005. Organic compounds in the environment: Atrazine sorption445 desorption hysteresis by sugarcane mulch residue. Journal of Environmental Quality 34, 325-335.

Strahm B.D., Harrison R.B. 2008. Controls on the sorption, desorption and mineralization of low-molecular-weight organic acids in variable-charge soils. Soil Science Society of America Journal 72, 1653-1664.

Ström L., Owen A.G., Godbold D.L, Jones D.L. 2001. Organic acid behavior in a calcareous soil: sorption reactions and biodegradation rates. Soil Biology \& Biochemistry 33, 21252133.

Tsakiroglou C.D., Ioannidis M.A. 2008. Dual-porosity modelling of the pore structure and transport properties of a contaminated soil. European Journal of Soil Science 59, 744761.

van Hees P.A.W., Johansson E., Jones D.L. 2008. Dynamics of simple carbon compounds in two forest soils as revealed by soil solution concentrations and biodegradation kinetics. Plant and Soi 310, 11-23.

van Hees P.A.W., Jones D.L., Godbold D.L. 2002. Biodegradation of low molecular weight organic acids in coniferous forest podzolic soils. Soil Biology \& Biochemistry 34, 12611272.

van Hees P.A.W., Vinogradoff S.I., Edwards A.C., Godbold D.L., Jones D.L. 2003. Low molecular weight organic acid adsorption in forest soils: effects on soil solution concentrations and biodegradation rates. Soil Biology \& Biochemistry 35, 1015-1026.

Wouterlood M., Cawthray G.R., Scanlon T.T., Lambers H., Veneklaas E.J. 2004. Carboxylate concentrations in the rhizosphere of lateral roots of chickpea (Cicer arietinum) increase during plant development, but are not correlated with phosphorous status of soil or plants. New Phytologist 162, 745-753. 
469 Zhang F.S., Ma J., Cao Y.P. 1997. Phosphorus deficiency enhances root exudation of low 470 molecular weight organic acids and utilization of sparingly soluble inorganic 471 phosphates by radish (Raghanus sativus L.) and rape (Brassica napus L.) plants. Plant $472 \quad$ and Soil 196, 261-264.

473 Zhang H., Selim H.M. 2005. Kinetics of arsenate adsorption - desorption in soils $474 \quad$ Environmental Science \& Technology 39, 6101-6108. 
Table 1

General properties of the three soils used in the organic acid sorption experiments.

\begin{tabular}{lcccc}
\hline & & Cambisol Ah & Podzol Ah & Podzol Bs \\
\hline $\mathrm{pH}\left(\mathrm{H}_{2} \mathrm{O}\right)$ & & $5.85 \pm 0.12$ & $4.95 \pm 0.16$ & $4.72 \pm 0.04$ \\
Organic matter & $\left(\mathrm{g} \mathrm{kg}^{-1}\right)$ & $12.9 \pm 1.2$ & $23.8 \pm 3.0$ & $10.3 \pm 0.5$ \\
Crystalline oxides & & & & \\
$\mathrm{Fe}$ & $\left(\mathrm{g} \mathrm{kg}^{-1}\right)$ & $1.41 \pm 0.05$ & $1.88 \pm 0.11$ & $1.85 \pm 0.02$ \\
$\mathrm{Al}$ & $\left(\mathrm{g} \mathrm{kg}^{-1}\right)$ & $1.43 \pm 0.06$ & $2.32 \pm 0.28$ & $1.49 \pm 0.29$
\end{tabular}

Amorphous oxides

$\begin{array}{rrrrrr}\mathrm{Fe} & \left(\mathrm{g} \mathrm{kg}^{-1}\right) & 5.0 & \pm 0.1 & 9.3 \pm 0.0 & 11.8 \pm 0.2 \\ \mathrm{Al} & \left(\mathrm{g} \mathrm{kg}^{-1}\right) & 1.6 \pm 0.0 & 2.7 \pm 0.0 & 3.6 \pm 0.1\end{array}$

Total (aqua regia)

$\begin{array}{lllllll}\mathrm{Al} & \left(\mathrm{g} \mathrm{kg}^{-1}\right) & 27.8 & \pm 0.6 & 25.7 & \pm 0.9 & 30.1 \pm 0.8 \\ \mathrm{Fe} & \left(\mathrm{g} \mathrm{kg}^{-1}\right) & 45.8 & \pm 0.5 & 49.2 \pm 0.5 & 57.9 \pm 0.3 \\ \mathrm{Ca} & \left(\mathrm{g} \mathrm{kg}^{-1}\right) & 1.91 & \pm 0.06 & 0.29 \pm 0.02 & 0.22 \pm 0.01\end{array}$

Values represent means $\pm \operatorname{SEM}(n=3)$.

Table 2

Sorption constants obtained by fitting the Barrow-modified Freundlich model to the experimental adsorption and desorption data

\begin{tabular}{lllll}
\hline \multicolumn{2}{c}{ Model parameters } & Cambisol & Podzol Ah & Podzol Bs \\
\hline Adsorption & $k_{\mathrm{ad}}$ & $4.782 \times 10^{-3}$ & $5.489 \times 10^{-3}$ & $2.155 \times 10^{-2}$ \\
& $n_{\mathrm{ad}}$ & 0.899 & 0.93 & 0.737 \\
& sse & $1.146 \times 10^{-3}$ & $2.646 \times 10^{-3}$ & $3.483 \times 10^{-3}$ \\
Desorption & $k_{\mathrm{de}}$ & 0.208 & & \\
& $n_{\mathrm{de}}$ & 0.159 & 0.127 & 0.298 \\
& sse & $3.203 \times 10^{-3}$ & $2.514 \times 10^{-3}$ & $9.823 \times 10^{-4}$ \\
Kinetic & & & & \\
model & & 0.545 & 1.378 & 3.046 \\
& $\Theta$ & $3.866 \times 10^{-4}$ & $1.226 \times 10^{-10}$ & $1.352 \times 10^{-13}$ \\
& sse & $9.734 \times 10^{-3}$ & $1.267 \times 10^{-2}$ & $8.454 \times 10^{-3}$ \\
\hline
\end{tabular}




\section{Figure Legends}

\section{Figure 1}

Adsorption and desorption isotherms with the corresponding kinetic data - experimental data and modelling results.

\section{Figure 2}

Phase portrait of the dynamic sorption model. Between the two stable steady states (ad- and desorption isotherm) lies an unstable steady states. Depending of the initial state $\left(c_{l}, c_{s}\right)$ either adsorption (in area B and D) or desorption (in area A and C) occurs.

Figure 3

Predicted solid-solution partitioning of $40 \mu \mathrm{M}$ citrate originated from a root exudation event in the Podzol Bs soil and the effect of consecutive exudation event $(+15 \mu \mathrm{M})$ (a) and the time course of changes in soil solution concentration induced by citrate sorption and the second exudation event (b).

Figure 4

Predicted solid-solution partitioning of $40 \mu \mathrm{M}$ citrate originated from a root exudation event in the Podzol Bs soil and the effect of microbial degradation (- $15 \mu \mathrm{M}$, no time dynamics of the mineralization process considered) (a) and the time course of changes in soil solution concentration induced by citrate sorption and microbial degradation (b). 


\section{Figure 1}

Adsorption and desorption isotherms with the corresponding kinetic data - experimental data and modelling results.

(a) Sorption Isotherms

Cambisol

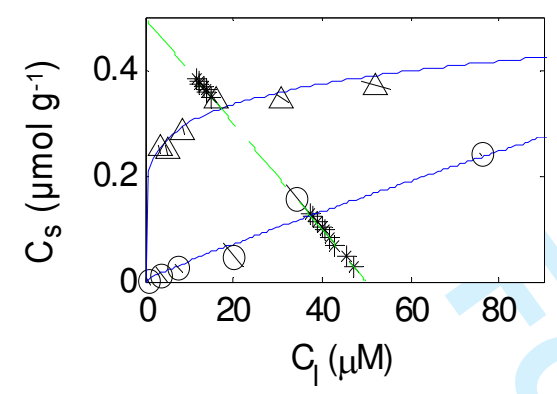

Podzol Ah

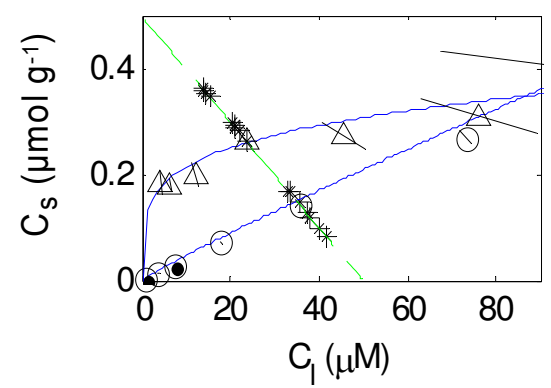

Podzol Bs

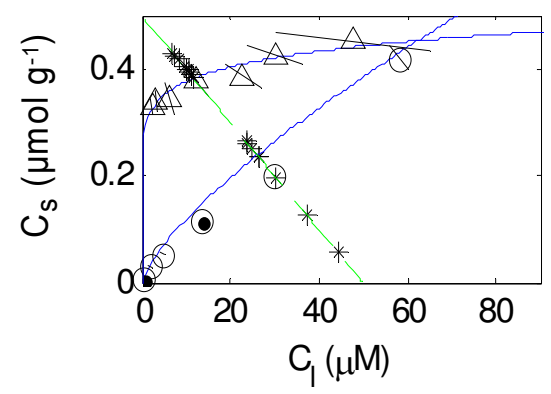

$\triangle \quad$ Desorption data

O Adsorption data

* Kinetic data

Model fit (b) Adsorption kinetics

Cambisol

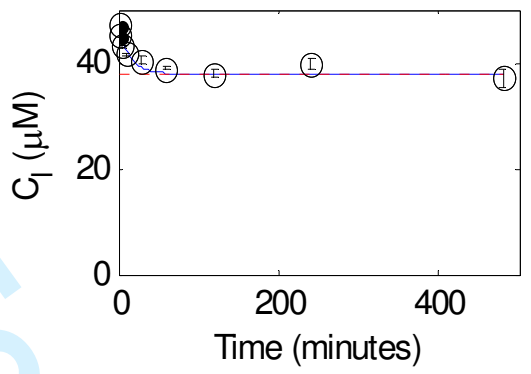

Podzol Ah

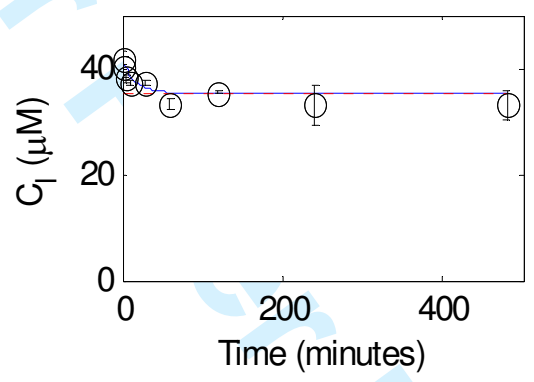

Podzol Bs

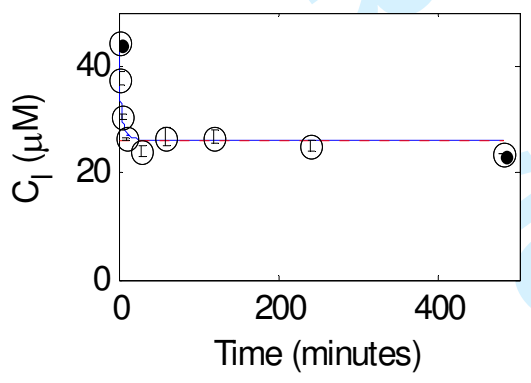

O Adsorption data

- Model fit

- - - Isotherm equilibrium (c) Desorption kinetics

Cambisol

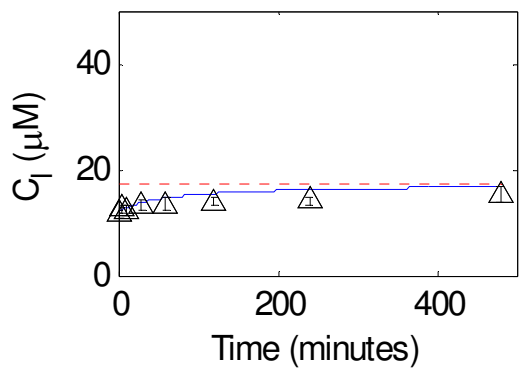

Podzol Ah

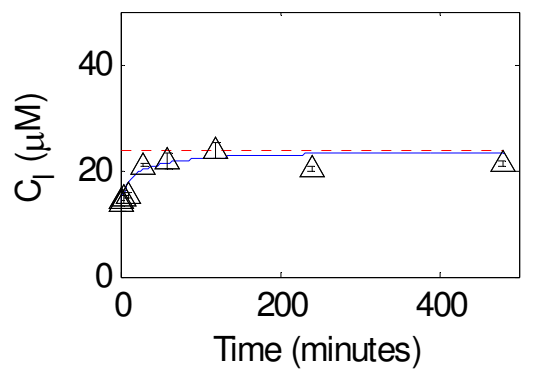

Podzol Bs

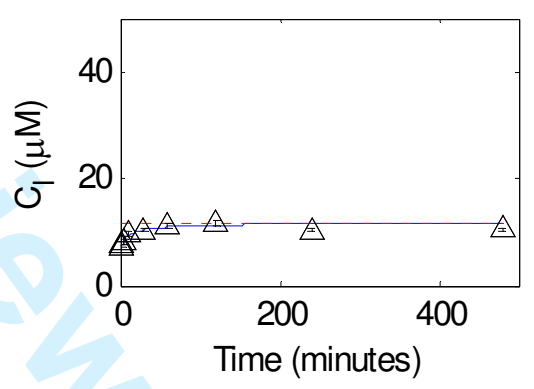

$\Delta$ Desorption data

- Model fit

- - - Isotherm equilibrium 


\section{Figure 2}

Phase portrait of the dynamic sorption model. Between the two stable steady states (ad- and desorption isotherm) lies an unstable steady states. Depending of the initial state $\left(c_{l}, c_{s}\right)$ either adsorption (in area B and D) or desorption (in area A and C) occurs.

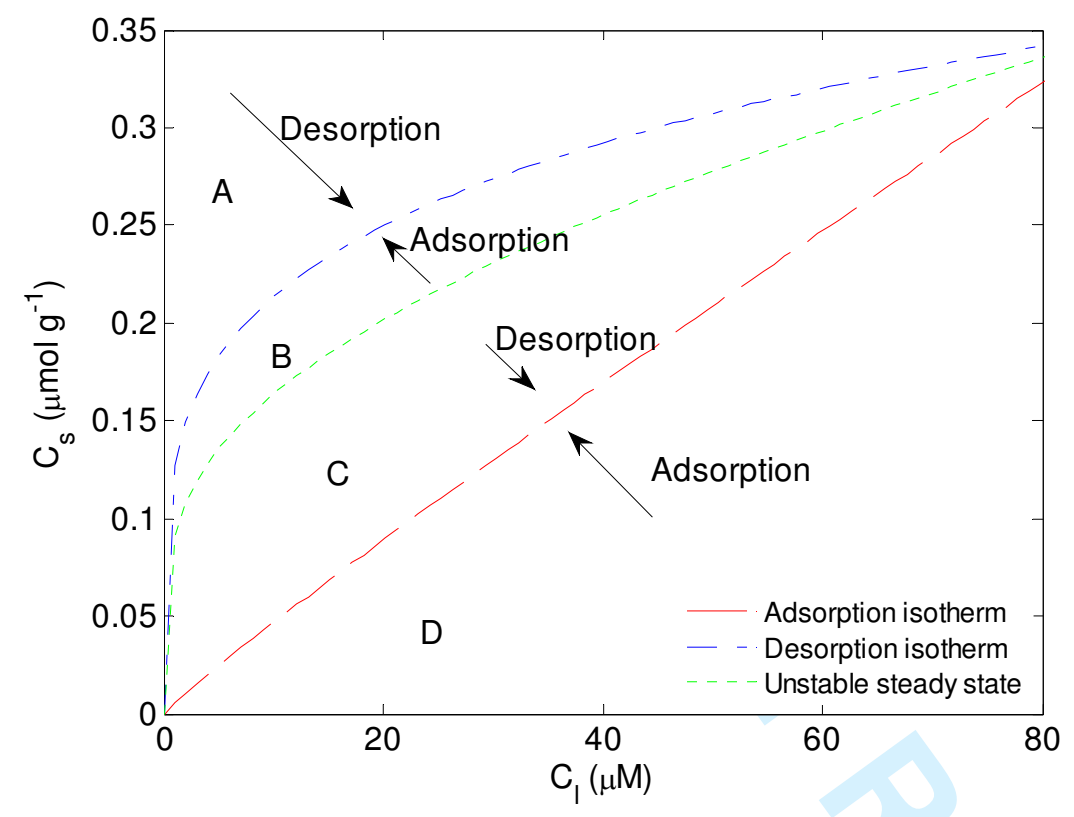




\section{Figure 3}

Predicted solid-solution partitioning of $40 \mu \mathrm{M}$ citrate originated from a root exudation event in the Podzol Bs soil and the effect of consecutive exudation event $(+15 \mu \mathrm{M})(\mathrm{a})$ and the time course of changes in soil solution concentration induced by citrate sorption and the second exudation event (b).

(a)

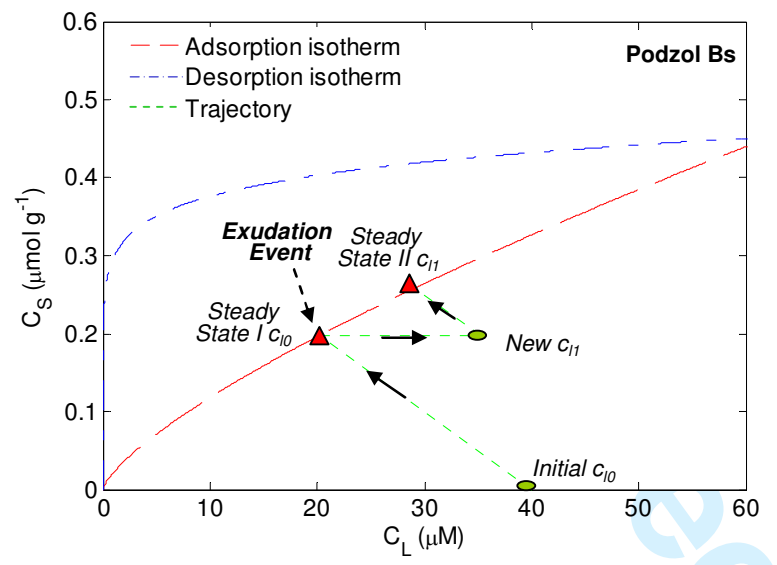

(b)

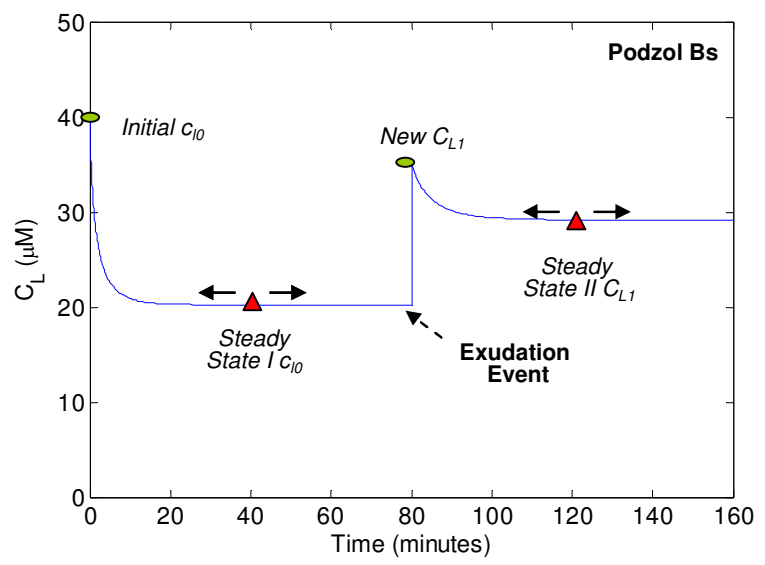


Figure 4

Predicted solid-solution partitioning of $40 \mu \mathrm{M}$ citrate originated from a root exudation event in the Podzol Bs soil and the effect of microbial degradation (- $15 \mu \mathrm{M}$, no time dynamics of the mineralization process considered) (a) and the time course of changes in soil solution concentration induced by citrate sorption and microbial degradation (b).

(a)

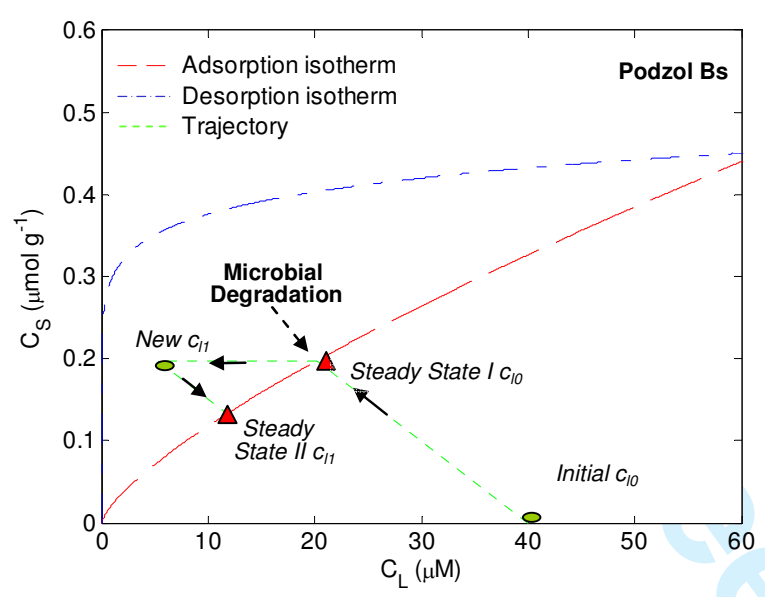

(b)

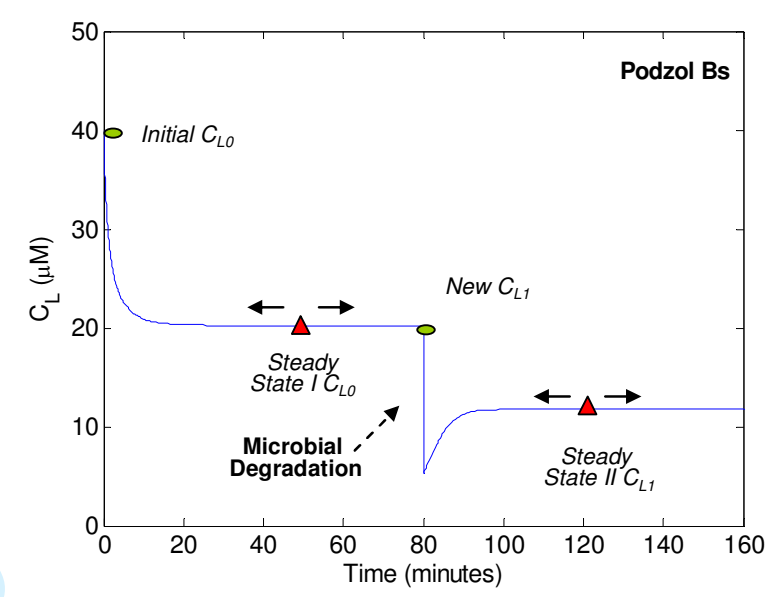





\section{RECENT REPORTS}

39/10 Local synaptic signaling enhances the stochastic transport of Newby motor-driven cargo in neurons

Bressloff

40/10 Convection and Heat Transfer in Layered Sloping Warm-Water

McKibbin Aquifer

Hale

Style

Walters

41/10 Optimal Error Estimates of a Mixed Finite Element Method for

Goswami Parabolic Integro-Differential Equations with Non Smooth Initial Data

Pani

Yadav

42/10 On the Linear Stability of the Fifth-Order WENO Discretization

Motamed

Macdonald

Ruuth

43/10 Four Bugs on a Rectangle

Chapman

Lottes

Trefethen

44/10 Mud peeling and horizontal crack formation in drying clay

Style

Peppin

Cocks

45/10 Binocular Rivalry in a Competitive Neural Network with Synaptic Depression

Kilpatrick

Bressloff

46/10 A theory for the alignment of cortical feature maps during development

Bressloff

Oster

47/10 All-at-Once Solution if Time-Dependent PDE-Constrained Optimisation Problmes

Stoll

Wathen

48/10 Possible role of differential growth in airway wall remodeling in asthma

Moulton

Goriely

49/10 Variational Data Assimilation Using Targetted Random Walks

Cotter

Dashti

Robinson

Stuart

50/10 A model for the anisotropic response of fibrous soft tissues using six discrete fibre bundles

Flynn

Rubin

Nielsen

51/10 STOCHSIMGPU Parallel stochastic simulation for the Systems Biology Toolbox 2 for MATLAB

Klingbeil

Erban

Giles

Maini

52/10 Order parameters in the Landau-de Gennes theory - the static Majumdar and dynamic scenarios

53/10 Liquid Crystal Theory and Modelling Discussion Meeting

Majumdar 
54/10 Modeling the growth of multicellular cancer spheroids in a bioengi-

Loessner neered 3D microenvironment and their treatment with an anticancer drug

Flegg

Byrne

Hall

Moroney

Clements

Hutmacher

McElwain

55/10 Scalar Z, ZK, KZK, and KP equations for shear waves in incompressible solids

Destrade

Goriely

Saccomandi

56/10 The Influence of Bioreactor Geometry and the Mechanical Environment on Engineered Tissues

Osborne

ODea

Whiteley

Byrne

Waters

$57 / 10$ A numerical guide to the solution of the bidomain equations of

Pathmanathan cardiac electrophysiology

Bernabeu

Bordas

Cooper

Garny

Pitt-Francis

Whiteley

Gavaghan

58/10 Particle-scale structure in frozen colloidal suspensions from small angle X-ray scattering

Spannuth

Mochrie

Peppin

Wettlaufer

$59 / 10 \quad$ Spin coating of an evaporating polymer solution

Munch

Please

Wagner

60/10 Stochastic synchronization of neuronal populations with intrinsic

Bressloff and extrinsic noise

Lai

61/10 Metastable states and quasicycles in a stochastic Wilson-Cowan

Bressloff model of neuronal population dynamics

Copies of these, and any other OCCAM reports can be obtained from:

Oxford Centre for Collaborative Applied Mathematics Mathematical Institute 24 - 29 St Giles' 
Oxford

OX1 3LB

England

www.maths.ox.ac.uk/occam 